\title{
The Combined Effects of Magnetic Asymmetry, Assembly and Manufacturing Tolerances on the Plasma Heat Load to the ITER First Wall
}

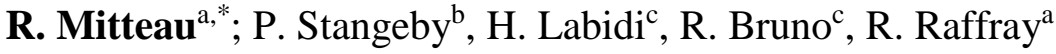 \\ $a$ - ITER Organization, Route de Vinon sur Verdon, 13115 St. Paul lez Durance, France \\ $b$ - Univ. of Toronto Institute for Aerospace Studies, 4925 Dufferin St, Toronto, M3H 5T6, Canada \\ c - Assystem France, 117 rue Jean Marie Jacquard, 84120 Pertuis, France
}

\begin{abstract}
The base plasma heat load on the ITER first wall results from the projection of the plasma scrape off layer power profile onto the shaped surface of the first wall panels. The actual heat load on the first wall is larger than the base heat load, due to enhancement factors such as design approximations, manufacturing and assembly errors, and deviations to the ideally toroidal magnetic field configurations. The heat load increase is accounted for through penalty coefficients, ranging individually from a few percent to $50 \%$. The probabilistic combination of individual penalty coefficients to give a global penalty coefficient is presented in the paper. The global penalty coefficient ranges from 1.37 to 2.44 , depending of the first wall row and plasma phase.
\end{abstract}

\section{Introduction}

The ITER first wall (FW) is the main protection of the ITER vessel against the plasma heat and particle fluxes. Owing to the modular construction of the ITER blanket, each blanket module is covered by an individual FW panel of dimensions about $1.5 \mathrm{~m}$ toroidally and $1 \mathrm{~m}$ poloidally. The ITER heat load specifications [1] indicates that parallel heat flux coming from the plasma scrape off layer (SOL) reaches the FW with a density ranging from 10 to 50 $\mathrm{MW} / \mathrm{m}^{2}$. Each FW panel is shaped, so that the panel edges are protected from the parallel heat flux either by shadowing or by placing them in a low heat flux zone, by taking benefit of the heat flux decay of the SOL. The FW panel shape results from a delicate optimisation [2], so that both edge load and surface heat load are balanced. The projected (engineering) heat load at the panel surface depends strongly on the surface shaping of the FW panels. The 
relevant scale here is the tile size (12 to $40 \mathrm{~mm}$ depending on the location), which matters for the integrity of the plasma facing component (armour bonding to the cooling circuit, heat exhaust through the cooling channels). This heat load determines the detailed design of the cooling circuits (hyper-vapotron or smooth channels, full copper or mixed steel/copper cooling circuits, coolant mass flow rate allocation, etc.). The design values for allowable projected heat flux density spans from 2 to $4.7 \mathrm{MW} / \mathrm{m}^{2}$ (Fig. 1), the panels with a higher heat load capacity being used in heavily loaded wall areas while those with a lower heat load capacity are used in regions only contacted by the very far scrape-off layer plasma (SOL).

The plasma SOL wets the FW with variable intensity during the different phases of the plasma discharge [1]. The plasma contact with the wall is mainly toroidal, which means that only one or two rows of FW panels have a high load for a given situation, a topology which is that of a toroidal limiter. Higher load density is expected (a) during start-up and ramp down phases, on the inner (row 3-4-5) or outer (rows 14 to 17) FW rows next to the midplane, when the plasma is being formed or extinguished, and the wall plays the role of a plasma limiter; (b) during diverted phases, where a large heat load may be deposited on the upper FW (rows 7-8-9) due to the presence of a secondary X-point at the top of the vessel, diverting hot flux surfaces that would otherwise reach the divertor [3]. The magnitude of the FW heat load is primarily determined by the shape of the magnetic surfaces (which determines the location of the main plasma wall interaction zone and its extension) and the SOL heat flux radial gradient. This first estimate of the heat flux density, the "base" level, is conveniently calculated through field line tracing and SOL power profile projection onto the actual shape of the plasma-facing components [4]. The result of such a calculation is illustrated in Fig. 2, for a limiter-type contact on a first wall panels of row 14.

The actual heat flux density onto the FW surface is also affected by other factors, which either increase or reduce the heat load relative to the base level. These factors include local design deviations from ideal-surface shape, manufacturing and assembly tolerances, and magnetic perturbations (toroidal field ripple, test blanket modules). Some of these factors can be variable depending upon the plasma conditions or location of the plasma contact to the wall (SOL width, plasma position) or are simply unknown at the design phase (manufacturing and assembly errors). A fully self-consistent inclusion of all possible perturbed heat load cases with all possible combination of perturbation factors would be impractical, given the number of parameters. Finding a priori the worst perturbed case is also impractical, because of the variety of the factors involved. A convenient way of handling this issue is to account 
for the various factors through heat load penalties. The penalty is simply a multiplication factor that conveniently accounts for some possible heat load perturbation events causing a modest heat load increase compared to the base heat load.

The heat load penalty is obtained by calculating two design situations : one with the perturbation and one without it. This test-case situation allows for a simple a-priori identification of the worst case situation. The resulting projected heat load on the first wall is extracted from the two calculations, and the penalty is obtained by taking the ratio of the two heat loads, respectively. This results in a number that is usually slightly above unity, or sometimes expressed as a percentage increase. It is a non-dimensional number.

The format of this paper does not allow for a full derivation of all the penalties. The scope is limited to quantifying the individual penalties and discusses briefly the main assumption to obtain those (section 2). Section 3 present how the penalties are combined together, toward deriving realistic penalty coefficients.

\section{Individual penalties}

\subsection{Design penalties}

The first wall shaping optimisation process [2] leads to an idealised smooth FW shape, maximizing the wetted area. The actual 'built to print design' requires simplifications (toroidal facets grouping several tiles on a same planar surface, poloidal chamfers) with respect to the smooth surface. The simplification increases locally the field line incidence angle to the tiles, hence the heat load. The penalty is set by design to 1.2 , which cascades into detailed requirements bounding the facet and chamfer sizes.

\subsection{Manufacturing penalties}

The manufacturing tolerances are set so that the heat load penalty is less than 1.1. Practically, this translates into a maximum tile angle deviation. There are other specific manufacturing tolerances (surface tolerance, finger to finger step, tile to tile step) which relates to the allowable edge heat load. This topic falls outside of the scope of this paper, because only the front heat load is addressed here.

\subsection{Assembly}

The assembly errors of the first wall are key contributors to heat load penalties. They affect the surface heat load in several ways. First, there is the radial positioning error with respect to 
the next neighbours, also called "short wave-length penalty error". A protruding panel receives a higher heat load than one aligned to its neighbours. This is because a few protruding panels do not modify significantly the scrape off layer thickness and position given the large number of panels in a row (18), [a full explanation is given in section 8 in Ref. 2]. Because of SOL invariance when few panels are misaligned, a protruding panel is exposed to a larger parallel heat flux, which translates to a larger projected heat load. In addition to this effect, the wetted area also increases due to the reduced shadowing by neighbour panels. This penalty is calculated as 1.5 on the outboard from the direct effect of the exponential increase of the parallel power in the scrape off layer (An increase factor of $\mathrm{e}^{5 / 12}, 5 \mathrm{~mm}$ being the design misalignment and $12 \mathrm{~mm}$ being the steepest outboard SOL decay length. The short wave penalty on the inboard is only 1.2, thanks to a longer power decay length of $50 \mathrm{~mm}$, for the same design step of $5 \mathrm{~mm}$. There is no misalignment penalty for the top FW panels which operate in diverted configuration (For diverted configuration, radial misalignment does not expose the panel to a higher heat flux density).

The second assembly penalty is the eccentricity between the average circular row of FW panels and the magnetic axis, also called long wave-length misalignment. This is also a radial positioning error, but the SOL (power width, position of the separatrix) is affected because of the larger number of panels involved. This penalty is calculated from power balance calculation, assuming the blanket module receives a power load which depends only on their position in the scrape off layer. The power balance calculation results in a sum of exponential factors, and happens to result in a factor of 1.45 . This is close to the short wave misalignment factor, although the mechanism of heat load increase is different. The eccentricity penalty is also 1.2 for the inboard rows based on the same calculation guideline.

Finally, a third assembly error is related to the angular positioning of the FW panel around the poloidal rotation axle. This mis-positioning increases the heat load on one side of the panel, and reduces it on the other side. This penalty is set to 1.1 .

\subsection{Magnetic perturbation}

The heat load on the FW panel is calculated for an ideal axisymmetric magnetic configuration. Toroidal magnetic field (TF) ripple, ELM control coils, and the presence of ferromagnetic material in the test blanket modules (TBM) deviates the magnetic field lines, which affect the incidence angle of the field lines on the panel, hence the heat load. The effect of the TF ripple is calculated as 1.16 for an uncorrected ripple. The TF ripple is 
mitigated by ferromagnetic inserts. The correction is optimised for a situation half way between half and full toroidal field. The penalty associated with the TF ripple is then estimated as 1.04 .

The penalty associated with the TBM is calculated as 1.2 , from a 3D map of the magnetic field including the TBM perturbation.

Finally, the heat load penalty associated with in vessel coils is set to zero, since these coils operates mainly in pulsed mode with a time scale of tenths of seconds or less which are much smaller than the thermal time constant of the plasma facing components (seconds). The time averaged effect of the in-vessel coils on the heat load can hence be neglected.

The heat load associated with the charge exchange neutrals and photon radiation is added $\left(0.35 \mathrm{MW} / \mathrm{m}^{2}\right.$ in diverted operation which is associated to high power operation - up to $500 \mathrm{MW}$, conservatively $0.25 \mathrm{MW} / \mathrm{m}^{2}$ in limiter operation where the plasma power is less than 7.5 MW.). While it is not really a penalty, it is still mentioned here since it is a contributor to a realistic estimation of the heat load to the first wall. Table 1 summarises the individual penalties, expressed in percentage.

\section{Combined penalties}

An approach that consists of piling up all penalties by successively multiplying all of them ('worst - worst case approach') would result in a large combined penalty, of up to a factor 5. This would not give a realistic estimate of the probable actual heat load. A more realistic accounting of these contributions involves a probabilistic approach, so that a better estimate of the FW heat load is obtained for the various possible combinations of factors. Probabilistic combination involves 'root mean square' combination of the individual penalties. This combination rule provides a more moderate combination of individual penalties when it is estimated that there is a small risk that the two penalties will occur at the same place and at the same time. It also accounts for the fact that some situations may come to an actual reduction of the heat load, so that a realistic combination involves positive and negative contributors. An even softer way to combine penalties is to take the largest one, which is appropriate when there is very low probability that the two penalties combine.

The combination laws are illustrated as an example, for the two main outboard assembly penalties. As described in section $2, p_{\text {step }}=1.5$ and $p_{\text {eccentricity }}=1.45$. Full combination gives

$p_{\text {combined step and eccentricity }}=2.18$, probabilistic combination gives $p_{\text {combined step and eccentricity }}=1.67$ 
(from $1.67=1+\sqrt{ }\left(0.5^{2}+0.45^{2}\right)$ ), and the exclusive combination gives $p_{\text {combined step and eccentricity }}$ $=1.5$.

The combination rules used for estimating the first wall heat load in ITER are as follows :

- The design penalties combines exclusively together, since they have already been estimated in combination (no reason for re-combining).

- The design penalty is usually present, hence it combines fully with the other penalties.

- The assembly and manufacturing penalties all combine in a probabilistic combination, since these are random errors which are not associated. For a set of 440 panels, there is a small probability that various worst case penalties combine at the same place.

- The magnetic penalties combine exclusively, since the TBM penalty was estimated including the ripple. (no reason for adding again the ripple)

- The magnetic penalties fully combined with the other ones since they are always present.

- Finally, the charge exchange and radiation heat load are added.

The combined penalties are shown in table 2. The table is labelled along the blanket rows, groupings the rows that have identical penalties : group 1 gathers rows 1-2-6 and have only far SOL plasma on the outboard, group 2 is rows 3-4-5 which have limiter contact on the inboard, group 3 are top panels, rows 7-8-9, group 4 is rows 10-11-12-13-18 which have only far SOL contact on the outboard, and finally group 5 is rows 14 to 17 having limiter contact on the outboard. The penalties range from 1.37 to 2.44 . The smallest penalty of 1.37 is obtained for the top panels which never operate in limiter mode, hence are less sensitive to misalignment errors. The largest penalty of 2.44 is obtained for the outboard rows in limiter operation, mainly due to the combination of large individual penalties. Table 2 also lists the projected heat load on the first wall rows, which is named "design heat load". The design heat load is the engineering heat load that is assumed to be deposited on the FW panels, with a reasonable confidence level, while attempting to include reasonable conservatism. This is the heat load that is taken into account for design activities, such as tile bonding qualification or design of the cooling circuits. The heat load is usually within the allowable of 2 and 4.7 $\mathrm{MW} / \mathrm{m}^{2}$, except for top panels. However, the excess heat load on the top panels is obtained for a situation at the smallest separatrix distance (DRsep $=4 \mathrm{~cm}$ ) in an unfavourable assembly case, a situation which is estimated to be low probability and may be alleviated by plasma operation at a larger DRsep. The penalty coefficients presented in this work may still 
contain some conservatism ; further conservatism could be reduced to some extent but this would require a significant additional effort.

\section{Conclusion}

Heat load penalties are a simple technique to account for unknown or variables parameters affecting the heat loads during the design phase. The individual penalties are described and quantified, ranging from a few percent up to $50 \%$. The combination rules are presented. The combined penalty spans from 1.37 to 2.44 for the ITER first wall, and needs to be accounted for when estimating heat load on ITER FW panels. Rows 3-4-5-7-8-9-14-15-16-17 receives larger heat load of 3.4 to $6.7 \mathrm{MW} / \mathrm{m}^{2}$ as the result of direct plasma contact, while rows 1-2-610-11-12-13-18 receive only far SOL heat load which are less than $2 \mathrm{MW} / \mathrm{m}^{2}$. The surface heat load on the first wall is usually within the allowable, except for top panels. The excess heat load on the top panels is obtained for a situation with the smallest separatrix distance (DRsep) in an unfavourable assembly case, a situation which is estimated to be of low probability and may be alleviated by plasma operation at a larger DRsep.

\section{Disclaimer}

The views and opinions expressed herein do not necessarily reflect those of the ITER Organization

\section{References}

[1] R.A. Pitts S. Carpentier, F. Escourbiac, et Al., Jour. Nucl. Mater. Volume 415, Issue 1, Supplement, 1 August 2011, Pages S957-S964, http://dx.doi.org/10.1016/j.jnucmat.2011.01.114

[2] Peter Stangeby, 2011 Nucl. Fusion 51103015 http://doi:10.1088/0029-5515/51/10/103015,

[3] R. Mitteau, P. Stangeby, C. Lowry et Al, , Jour. Nucl. Mater., Volume 415, Issue 1, Supplement, 1 August 2011, Pages S969-S972, http://dx.doi/10.1016/j.jnucmat.2010.10.070

[4] R. Mitteau, P. Stangeby, Journal of Nuclear Materials, Volumes 390-391, 15 June 2009, Pages 1022-1025, http://dx.doi.org/10.1016/j.jnucmat.2009.01.270 


\section{Figure captions}

Fig. 1 : Blanket rows

Fig. 2 : A First wall panel for row 14 and calculated base heat load for a plasma heat power of 7.5 MW, power decay length of $12 \mathrm{~mm}$, magnetic field line pitch of 0.154 and minor radius of $3 \mathrm{~m}$.

Table 1 : Individual heat load penalties on the ITER first wall

Table 2 : Combined heat load penalties on the ITER first wall 
Figure 1

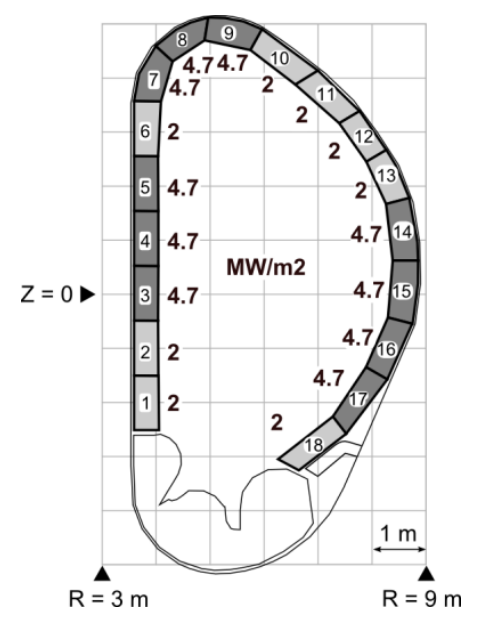


Figure 2
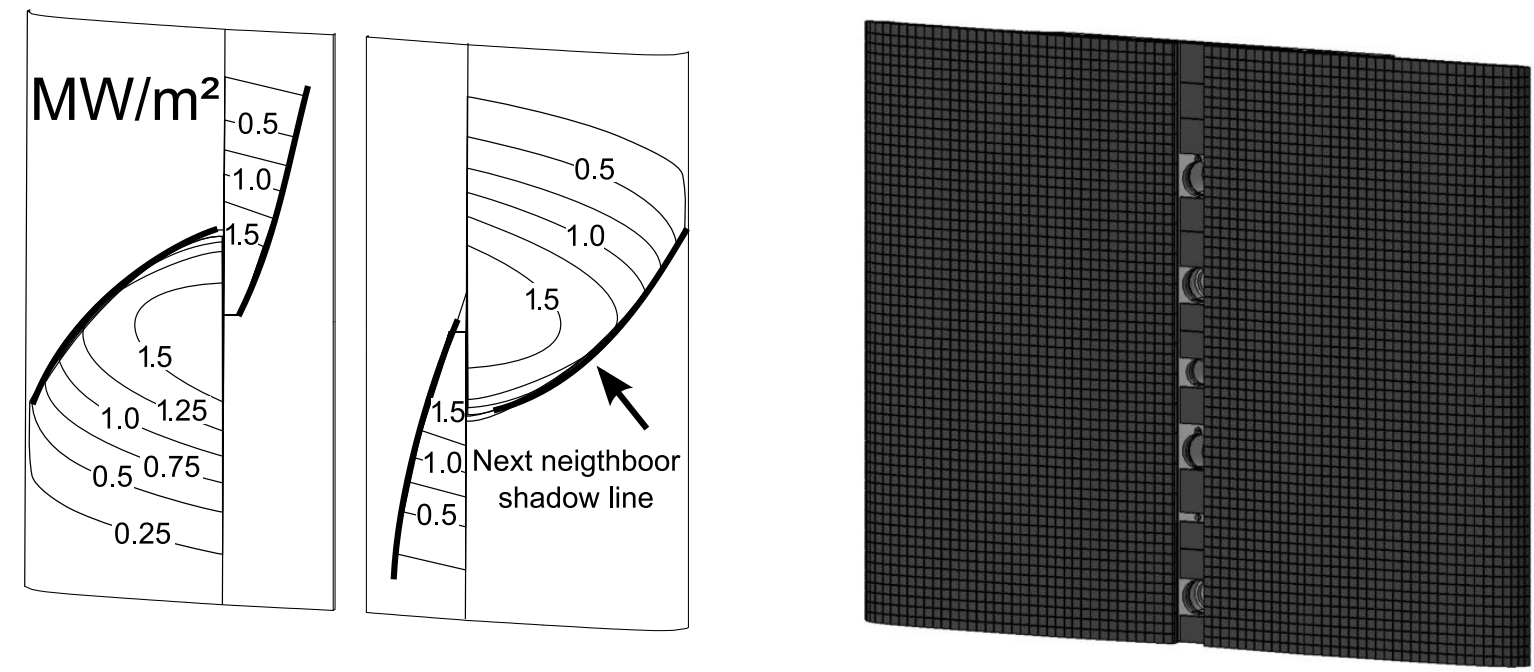
Table 1 :

\begin{tabular}{|c|c|c|}
\hline & Cause for the penalty & penalty \\
\hline \multirow[t]{2}{*}{ Design } & Facets & $+20 \%$ \\
\hline & Poloidal chamfering & $\begin{array}{l}+20 \% \text { (inboard and } \\
\text { top) }\end{array}$ \\
\hline Manuf. & Tile angular error & $+10 \%$ \\
\hline \multirow[t]{3}{*}{ Assembly } & Radial step & $\begin{array}{l}+50 \% \text { (outboard) } \\
+20 \% \text { (inboard) } \\
0 \text { (top) }\end{array}$ \\
\hline & Eccentricity & $\begin{array}{l}+45 \% \text { (outboard) } \\
+20 \% \text { (inboard) } \\
0 \text { (top) }\end{array}$ \\
\hline & Angular error & $+10 \%$ \\
\hline \multirow[t]{3}{*}{$\begin{array}{l}\text { Magnetic } \\
\text { deviations }\end{array}$} & Toroidal field ripple & $\begin{array}{l}+4 \% \text { (Outboard } \\
\text { only) }\end{array}$ \\
\hline & $\begin{array}{l}\text { Error field by Test } \\
\text { blanket Module }\end{array}$ & $\begin{array}{l}+20 \% \\
\text { (rows 13-16) }\end{array}$ \\
\hline & ELM, VS & 0 \\
\hline
\end{tabular}


Table 2

\begin{tabular}{|c|c|c|c|c|c|c|c|c|c|c|}
\hline & \multicolumn{4}{|c|}{ Limiter operation } & \multicolumn{4}{|c|}{ Divertor operation } & \multirow[b]{2}{*}{$\begin{array}{c}\text { Design } \\
\text { heat } \\
\text { load } \\
\left(\mathrm{MW} / \mathrm{m}^{2}\right)\end{array}$} & \multirow[b]{2}{*}{$\begin{array}{l}\text { Allowable } \\
\text { heat loao } \\
\left(\mathrm{MW} / \mathrm{m}^{2}\right)\end{array}$} \\
\hline & $\begin{array}{c}\text { Base heat } \\
\text { load } \\
\left(\mathrm{MW} / \mathrm{m}^{2}\right)\end{array}$ & Penalty & $\left(\begin{array}{c}\mathrm{Cx}+ \\
\mathrm{Rad} \\
\left(\mathrm{MW} / \mathrm{m}^{2}\right)\end{array}\right.$ & $\begin{array}{l}\text { Max heat } \\
\text { load limiter } \\
\left(\mathrm{MW} / \mathrm{m}^{2}\right)\end{array}$ & $\begin{array}{c}\text { Base heat } \\
\text { load } \\
\left(\mathrm{MW} / \mathrm{m}^{2}\right)\end{array}$ & Penalty & $\begin{array}{c}\mathrm{CX}+ \\
\mathrm{Rad} \\
\left(\mathrm{MW} / \mathrm{m}^{2}\right)\end{array}$ & $\begin{array}{l}\text { Max heat load } \\
\text { diverted op. } \\
\left(\mathrm{MW} / \mathrm{m}^{2}\right)\end{array}$ & & \\
\hline $\begin{array}{c}\text { Inboard rows } \\
1-2-6\end{array}$ & 1.00 & 1.58 & 0.25 & 1.83 & 1.00 & 1.58 & 0.35 & 1.93 & 1.9 & 2.0 \\
\hline $\begin{array}{c}\text { Inboard rows } \\
3-4-5\end{array}$ & 2.00 & 1.58 & 0.25 & 3.41 & 1.00 & 1.58 & 0.35 & 1.93 & 3.4 & 4.7 \\
\hline $\begin{array}{c}\text { Top panels } \\
7-8-9\end{array}$ & \multicolumn{4}{|c|}{ No significant heat load } & 4.60 & 1.37 & 0.35 & 6.65 & 6.7 & 4.7 \\
\hline $\begin{array}{l}\text { Outboard rows } \\
10-11-12-13-18\end{array}$ & 0.20 & 2.11 & 0.25 & 0.67 & 0.80 & 2.11 & 0.35 & 2.04 & 2.0 & 2.0 \\
\hline $\begin{array}{c}\text { Outboard rows } \\
14-15-16-17\end{array}$ & 1.56 & 2.44 & 0.25 & 4.05 & 0.70 & 2.44 & 0.35 & 2.06 & 4.1 & 4.7 \\
\hline
\end{tabular}

